\title{
The evolution of endovascular electroencephalography: historical perspective and future applications
}

\author{
Roberta K. Sefcik, BHA, ${ }^{1}$ Nicholas L. Opie, PhD, ${ }^{2}$ Sam E. John, PhD, MS, ${ }^{2}$ \\ Christopher P. Kellner, MD, ${ }^{3}$ J Mocco, MD, MS, ${ }^{3}$ and Thomas J. Oxley, MBBS ${ }^{2,3}$

\begin{abstract}
Departments of ${ }^{1}$ Medical Education and ${ }^{3}$ Neurosurgery, Icahn School of Medicine at Mount Sinai, New York, New York; and ${ }^{2}$ Vascular Bionics Laboratory, Department of Medicine, Royal Melbourne Hospital, The University of Melbourne, Victoria, Australia
\end{abstract}

\begin{abstract}
Current standard practice requires an invasive approach to the recording of electroencephalography (EEG) for epilepsy surgery, deep brain stimulation (DBS), and brain-machine interfaces (BMIs). The development of endovascular techniques offers a minimally invasive route to recording EEG from deep brain structures. This historical perspective aims to describe the technical progress in endovascular EEG by reviewing the first endovascular recordings made using a wire electrode, which was followed by the development of nanowire and catheter recordings and, finally, the most recent progress in stent-electrode recordings. The technical progress in device technology over time and the development of the ability to record chronic intravenous EEG from electrode arrays is described. Future applications for the use of endovascular EEG in the preoperative and operative management of epilepsy surgery are then discussed, followed by the possibility of the technique's future application in minimally invasive operative approaches to DBS and BMI.

http://thejns.org/doi/abs/10.3171/2016.3.FOCUS15635
\end{abstract}

KEY WORDS endovascular; intraarterial; electroencephalography; epilepsy; deep brain stimulation; brain-machine interface

$\mathrm{D}$ IRECT recording and stimulation of the brain has been used for decades as a method to investigate neural function and treat conditions such as intractable epilepsy ${ }^{30}$ and Parkinson's disease. ${ }^{8}$ The current surgical methods for accessing deep brain structures, however, require invasive open brain surgery: removing sections of skull in order to insert electrode arrays. While these invasive techniques have shown potential for deep brain stimulation (DBS) and brain-machine interface (BMI) technologies, access to the deep brain structures requires the traumatic penetration of electrodes directly into brain tissue. Access to deep brain structures can also be achieved via minimally invasive techniques by utilizing the cerebrovascular system as a conduit. ${ }^{31}$ The measurement and recording of neural information from wires, catheters, and stent-electrode arrays have demonstrated that the neural signals recorded from within a blood vessel are comparable to those obtained using invasive methods..$^{22}$ This article aims to offer a historical perspective on the development of endovascular electroencephalography (EEG) and discusses possible future applications of endovascular EEG in minimally invasive neurosurgery in the fields of epilepsy, DBS, and BMI.

\section{Technical Progress: A Historical Timeline}

The following timeline demonstrates the technical developments achieved in the recording of endovascular EEG from the first recordings in the early $1970 \mathrm{~s}^{23}$ to the most recent recordings in 2015.22 The technical progress in device development, from wire electrodes to nanowire and catheter electrodes and finally stent-electrodes (stentrodes), is described.

\section{Wire Recordings}

Experimentation with endovascular EEG recording devices began with the development and use of wire electrodes in the arterial system. The first device built on a 
magnetically guided catheter originally developed by Driller et al. for flexible cerebrovascular catheterization. ${ }^{7}$ Penn et al. modified this device to record the first endovascular EEG in humans in 1973. The device comprised a stainless steel wire with a helical inner spring coated in silicone and Teflon attached to a platinum-cobalt magnet that served as the electrode. The emergence of the spring from the wire also served as an electrode to enable bipolar recordings. This group noted that the endovascular recording contained electrocardiographic artifacts, but these artifacts were reduced as the electrode advanced proximally. Penn and colleagues' recordings in baboons revealed that endovascular EEG measurements had a higher amplitude when compared with extracranial EEG..$^{23}$

The following studies expanded on the work of Penn et $\mathrm{al}^{23}$ in performing endovascular arterial recordings. Reuter et al. performed arterial endovascular EEG with a wire electrode in baboons in 1989 and recorded somatosensory evoked potentials that were undetectable by surface EEG. ${ }^{25}$ Zeitlhofer et al. used the arterial endovascular approach in 1990 and had a larger sample size than Penn et al., ${ }^{23}$ with endovascular recordings in 8 humans. ${ }^{37}$

Unipolar wire electrodes were used to record arterial endovascular EEG throughout most of the 1990s. Nakase et al. expanded on previous work using a stainless steel Seeker Lite-10 wire and a Unibody Tracker-10 catheter ${ }^{20,21}$ with a single platinum electrode tip. ${ }^{21}$ This group performed endovascular recordings in 2 patients in 1993 and succeeded in detecting an interictal spike in both brain hemispheres. ${ }^{20}$ In experiments performed 2 years later, Nakase et al. performed intraarterial EEG in 28 patients and noted that intraarterial EEG recordings were consistent with subdural electrodes $(n=3)$, and the voltages of the intraarterial EEG recordings were 2 to 5 times that of extracranial EEG. The limitations of these experiments included that the recording device could not be used chronically and the recordings were only interictal and over a limited time and space. ${ }^{21}$

Stoeter et al..$^{28}$ used an endovascular arterial approach similar to that of Nakase et al., ${ }^{20,21}$ with slight modifications in the recording device. Stoeter et al. used a Seeker-10 guidewire that was coated in Polytef, a Tracher-18 microcatheter (Target Therapeutics), and a single platinum electrode. This group recorded EEG $(n=11)$ and evoked potentials $(n=12)$ in 23 patients undergoing embolization of brain tumors. ${ }^{28}$ These measurements were confirmed by extracranial electrodes and demonstrated an endovascular EEG potential that was 2 to 4 times the size of the extracranial electrodes, which was attributed to the direct communication of the electrode with the surface of the temporal lobe. A small percentage (27\%) of patients did not exhibit this relationship, which was observed to be due to the placement of the electrodes outside of the meningiomas. This relationship ceased when the electrode was placed inferior to the cranial base. Furthermore, EEG recordings from other territories (e.g., brainstem, parietal and frontal lobes) failed to show differences in amplitude when compared with extracranial electrodes. Endovascular recordings of the somatosensory evoked potentials demonstrated similar amplitudes to the extracranial measurements, while auditory evoked potentials had higher amplitudes when measured proximally compared with extracranial measurements. ${ }^{28}$

Boniface and Antoun also used a wire electrode technique to perform arterial endovascular EEG, but the device used differed in that it was a tapered, stainless steel, standard 14 steerable guidewire (Target Therapeutics) that was sprayed with silicone and covered in Teflon with a single platinum coil electrode. This group performed endovascular EEG in 2 patients undergoing carotid amytal assessment for intractable complex partial seizures in 1997 , and they recorded epileptiform spikes that were consistent with extracranial electrodes in 1 patient. ${ }^{4}$ Similar to the recordings of Penn et al., ${ }^{23}$ Boniface and Antoun also demonstrated the presence of pulse artifacts. ${ }^{4}$

At the end of the 1990s, García-Asensio et al. recorded the last arterial endovascular wire recordings using a stainless steel Seeker-10 microguidewire without a coating, Jetstream 10 and Tracker 10 catheters, and a single platinum electrode. This group experimented in $30 \mathrm{pa}-$ tients, producing 64 recordings that were performed in conjunction with surface EEG in 1999. García-Asensio et al. noted that the recordings contained the least amount of artifacts when they exposed only $1 \mathrm{~cm}$ of the electrode tip. Furthermore, the intraarterial recordings had 4 to 5 times the voltage of the surface recordings. The authors also administered valproic acid to 7 patients while simultaneously recording intraarterial EEG and found either the suppression or disappearance of epileptiform discharges. Using clinical epileptic crisis as the standard reference, the following statistical parameters were determined to describe intraarterial EEG: sensitivity, $93.33 \%$; specificity, $80 \%$; positive predictive value, $82.35 \%$; and negative predictive value, $92.3 \% .^{9}$

The application of the wire electrode technique in 1997 expanded both its use in the vascular approach (venous vs arterial) and the length of recording time. Mikuni et al. used a stainless steel Seeker Lite-10 wire (Target) coated in polymer with a platinum electrode to detect endovascular EEG from the cavernous sinus in 7 patients. Extracranial EEG $(n=6)$ and electrocorticography $(n=$ 4) confirmed the endovascular recordings. The venous endovascular approach detected EEG changes that were undetectable by the extracranial method, and the invasive electrocorticographic measurements acquired from a subdural array were consistent with the endovascular results. Mikuni et al. also demonstrated a predictive relationship between cavernous sinus recordings, revealing undetected EEG measurements by extracranial recordings and the presence of epileptogenic foci in the mesial temporal lobe as opposed to the lateral temporal lobe. In these experiments, this group secured the wire to the cervical skin, and, for the first time, continued recording EEG for 3 to 40 hours following angiography $(n=5) .{ }^{19}$

Kunieda et al..$^{16}$ expanded on the work of Mikuni et al. ${ }^{19}$ in terms of the detection of endovascular EEG from the cavernous sinus and the superior petrosal sinus and the length of the recording time. Kunieda et al. used a stainless steel Seeker Lite-10 wire (Target) coated in polymer with a single platinum electrode and detected $80 \%$ of ictal foci in patients $(n=5)$ with wires implanted for 4 to 75 hours..$^{16}$ As mentioned in previous studies, ${ }^{4,23}$ this group 
reported pulse artifacts in the recordings. The work by Kunieda et al. was limited by the patients' movements in the postanesthesia monitoring period, as patients were at risk of both wire breakage and inferior recordings. ${ }^{16}$

There were significant advances in endovascular wire recordings from the early 1970s through the late 1990s. At this point in time, recordings were usually from a single unipolar electrode, $4,9,16,19,21,28$ although the first endovascular EEG recording employed 2 electrodes for bipolar recording. ${ }^{23}$ Experimentation was translated from baboons ${ }^{23,25}$ to humans, ${ }^{4,9,16,19-21,23,28,37}$ and the sample sizes increased from the single recording in humans in $1973^{23}$ to recordings in 30 patients in 1999. ${ }^{9}$ From the first endovascular EEG recording in $1973,{ }^{23}$ there were also significant advances in both the ability to record from a venous approach and continue recordings outside of the angiography procedure..$^{16,19}$

\section{Nanowire Recordings}

Although others were still using standard wires at this time, ${ }^{9,16}$ there were advances in technology in 1998 with the development of microwires. Ishida et al. used a microwire in their intraarterial EEG recordings in 2 patients with simultaneously recorded extracranial EEGs. This group found that the intraarterial method was more sensitive in detecting interictal changes than the extracranial method, and the intraarterial approach was confirmed by the findings of chronic intracranial modalities..$^{14}$

Llinás et al. ${ }^{17}$ expanded on the work of Ishida et al ${ }^{14}$ in the use of microwire arrays, but their experiments were extracranial. Llinás et al. used 4 iron and platinum wires that were only $20 \mu \mathrm{m}$ in diameter to record EEG from the peripheral vascular/nervous system. This group also used an endovascular nanoelectrode to record EEG from the spinal cord, described the first nanowire array that consisted of 2 to 4 platinum black nanoelectrodes, and investigated the development of nanowires made of a conducting polymer. Building on this work, Llinás et al. devised a system in which multiple nanowires could hold millions of nanoprobes and measure EEG at the level of a single neuron. This group proposed that the device could be guided endovascularly to the brain and would arrange into a "bouquet" distribution within the brain's arterial/venous network. Despite the extent of brain territory that could be measured simultaneously with this method, Llinás et al. argued that due to the minuscule size of the instrument, physiological brain activity would not be altered. ${ }^{17}$

Extracranial experimentation with nanowires continued in 2009 with the work of Watanabe et al., who described the development and use of a Wollaston wire insulated with polymer and a micrometer platinum black electrode for recording endovascular EEG from the spinal cord of frogs. The endovascular nanowire EEG recording in this experiment was consistent with a silver spinal cordsurface electrode. ${ }^{34}$

From the late 1990 s to the early 2000 s, there was increasing miniaturization in recording wires. This allowed for the deployment of multiple wires in the form of wire arrays..$^{17}$ Despite the substantial progress in micro/nanowire technology, nanowire arrays have yet to be deployed in humans.

\section{Catheter Recordings}

In addition to the increasing miniaturization of wire electrodes in $1998,{ }^{14}$ a new technology for recording endovascular EEG was developed at the same time: the catheter electrode recording device. Thömke et al. demonstrated that a catheter (PathfinderTM) with 8 electrode pairs, which could simultaneously record from 16 locations in the brain (within a 72-mm distance), could be used to record endovascular EEG in humans..$^{32}$

Bower et al. advanced the catheter recording technique in 2013 with the inclusion of more electrodes and the first venous catheter recording. This group used 16 microelectrodes contained within a 4-contact depth electrode for the intravenous recording of EEG changes induced by penicillin and cortical electrical stimulation in pigs that underwent craniectomy for catheter placement in the superior sagittal sinus. The intravenous recordings were consistent in amplitude with simultaneously recorded subdural electrodes, and the intravascular method was successful in providing the location of seizure activity. Although Bower et al. advanced the catheter technique for its use in the superior sagittal sinus, the use of craniectomy for catheter placement limits the clinical utility of this demonstration as a future minimally invasive therapy. ${ }^{5}$

\section{Stentrode Recordings}

Endovascular EEG recording technology was advanced in terms of device design, location of device deployment, and the ability to chronically record endovascular EEG in 2016 with the development of the stentrode, which is pictured in Fig. 1. Oxley et al. determined through MRI analysis of 50 patients that the human superficial cortical veins and superior sagittal sinus, with intraluminal diam-

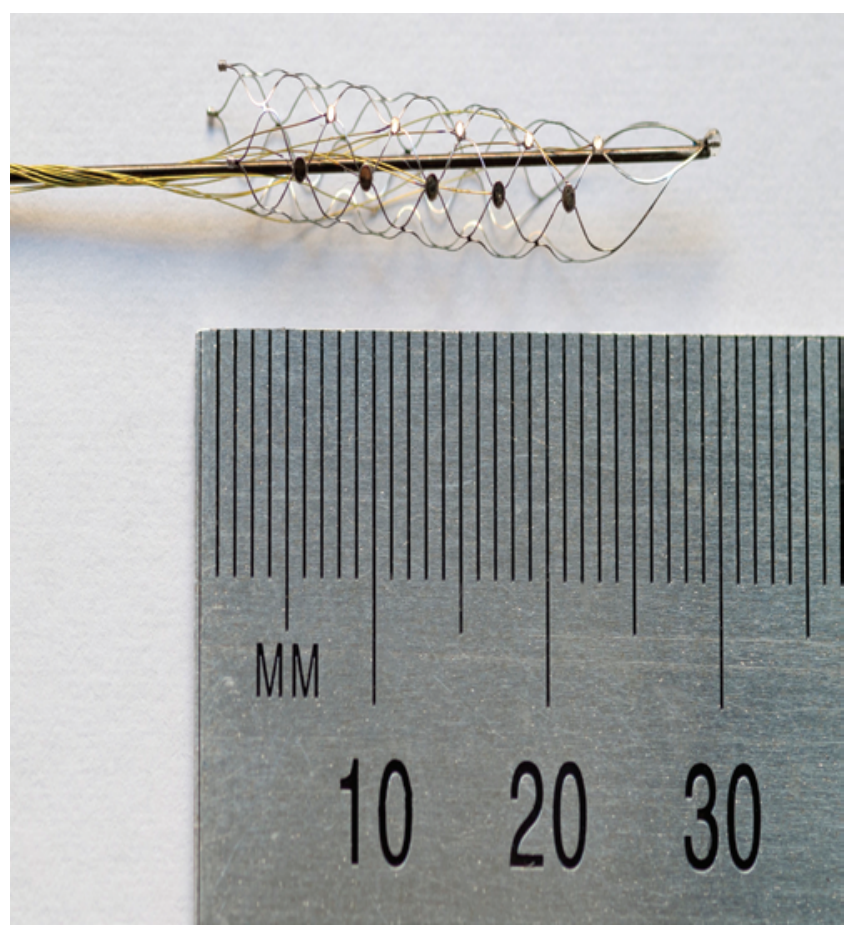

FIG. 1. A self-expanding stentrode is pictured adjacent to a ruler. 
eters of 2 to $8 \mathrm{~mm}$, were sufficient conduits for measuring neural activity from the sensorimotor cortex. Since the superior sagittal sinus in sheep is comparable to the central sulcal vein in humans, sheep were used to develop the animal model. Oxley et al. used a self-expanding stentrode array that was implanted in a superficial cortical vein via catheter angiography in order to chronically record neural activity (recordings up to 190 days) from the motor cortex in sheep and compared the recordings to both subdural and epidural surface arrays. The endovascular approach was comparable to the performance of the epidural array, but the performance of the subdural array was slightly superior. A pilot study resulted in the development of an epileptic seizure that was overcome by intravenous diazepam in 1 sheep, and repetitive neck movements by the freely moving sheep resulted in wire fatigue. There were also chewing muscle artifacts present in the recordings. ${ }^{22}$ Unlike the superior sagittal sinus recordings obtained by Bower et al., 5 the animals in the experiments conducted by Oxley et al. did not undergo craniectomy, thereby increasing the clinical utility of this approach as a minimally invasive therapy. Furthermore, the demonstration of the ability to chronically record endovascular EEG in the proximity of the sensorimotor cortex allows for possible applications in DBS and BMI, although further investigations of chronic recordings from endovascular stentrodes in humans are needed. ${ }^{22}$

\section{Discussion}

Endovascular recording devices have advanced from wire recordings ${ }^{4,9,16,19-21,23,25,28,37}$ to micro/nanowire recordings, ${ }^{14,17,34}$ to catheter recordings, ${ }^{5,32}$ and most recently stentrode recordings, ${ }^{22}$ as depicted in Fig. 2. Electrode arrays were developed from the increasing miniaturization in recording wires and electrodes ${ }^{17}$ and the development of catheter and stent-electrode recording technology, ${ }^{5,22,32}$ as illustrated in Table 1. The advances in endovascular recording, as summarized in Fig. 3, have made it possible to obtain increasing amounts of information about neural activity from the endovascular environment. Recording sites have also increased with the ability to record from the venous system..$^{5,16,19,22}$ Since the superior sagittal sinus is located superficial to the sensorimotor cortex, and the ability to chronically record endovascular EEG in freely moving animals has been demonstrated, ${ }^{22}$ there are possible future applications of the endovascular approach to EEG in BMI. The endovascular approach to EEG recording also promises greater safety for neural interfaces, as evidenced by the work of García-Asensio et al. who, with the largest human sample size in this review, reported no side effects or adverse events in their patients with up to 3 years of follow-up. ${ }^{9}$ In contrast, the subdural grid electrodes, to which many of the reviewed studies compared their endovascular recordings, $, 19,21,22$ were shown by Hamer et al. to have a complication rate of $26.3 \%$ (52 of 198 monitoring sessions).$^{11}$ While endovascular EEG offers an advantageous safety profile, risks should not be overlooked, such as the transient, but tolerable, headaches and retrobulbar pain as reported in the experiments of Mikuni et al. (such headaches were excluded from the study by Hamer et al. $\left.{ }^{11}\right) .{ }^{19}$

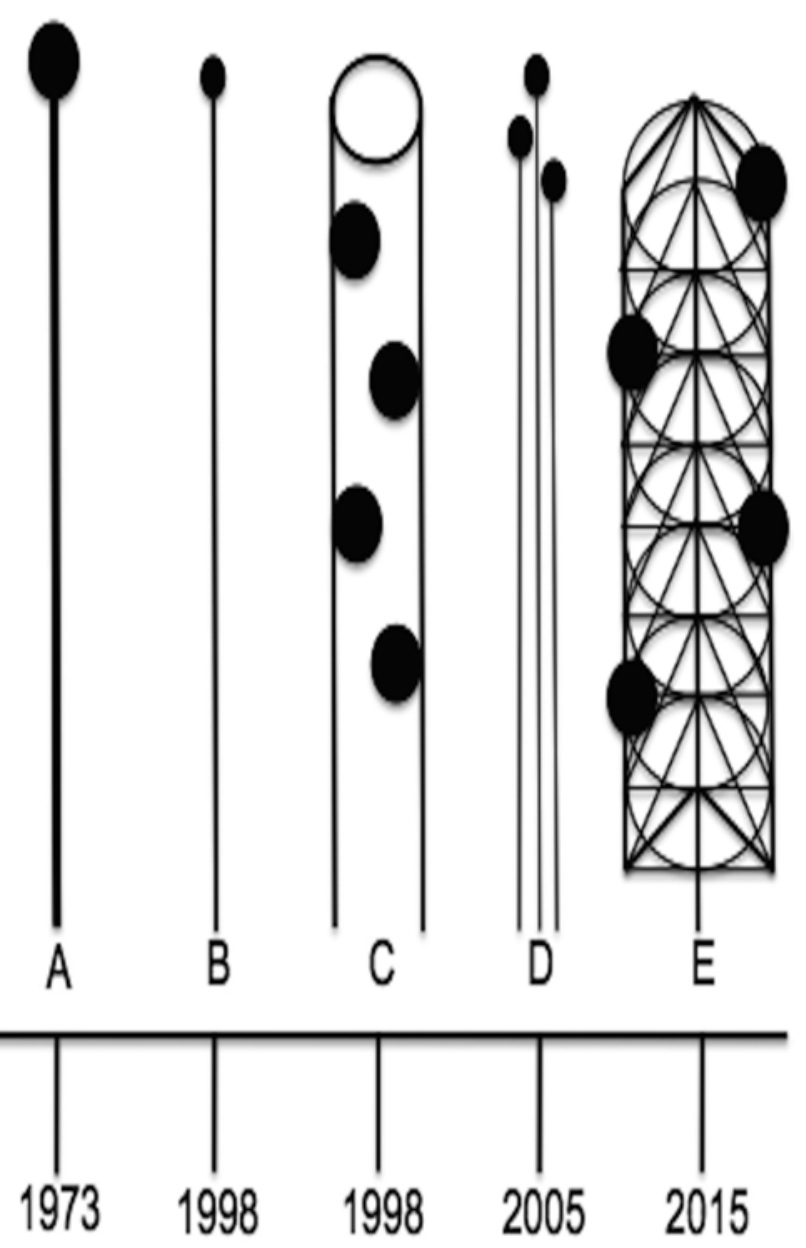

FIG. 2. Historical timeline depicting the changes in the structures of endovascular electrode devices. Schematic depiction of intravascular electrode array designs from 1973 to 2015 showing a guidewire with an electrode (black circle) placed on the proximal end (A), a microwire with an electrode (B), electrodes mounted on the outside of a catheter (C), a nanowire electrode array (D), and electrodes mounted on a selfexpanding stent (E).

\section{Future Applications}

\section{Epilepsy}

As previously discussed, the possibilities for the application of an endovascular approach to the recording of EEG are numerous in the field of epilepsy surgery. Current evaluative methods that are efficacious for the detection of seizure foci employ techniques such as the use of intracranial epidural arrays; however, this approach requires craniotomy. ${ }^{10}$ Likewise, intracranial depth electrode implantation aids in the detection of epileptogenic focus surgical margins, but requires invasive bur holes. ${ }^{27}$ The endovascular approach could be applied preoperatively for the minimally invasive localization of seizure foci ${ }^{5,16,19,22}$ and possibly the determination of resection margins. It is also possible that the endovascular approach could be employed for the surgical treatment of epileptogenic foci via endovascular ablation, as Ammerman et al. described a case report of a patient who became seizure free while receiving antiepileptic drugs following a stroke in the ter- 
TABLE 1. Historical timeline of recording electrode device specifications

\begin{tabular}{|c|c|c|c|c|c|c|}
\hline Authors \& Year & Device Category & Species (n) & Location & No. of Electrodes & Electrode Size & Electrode Material \\
\hline Penn et al., 1973 & Wire & Human (1), baboon & Arterial & 2 & $\begin{array}{l}\text { Length: } 1.5 \mathrm{~mm} \text {; diameter: } \\
\quad 0.6 \mathrm{~mm}\end{array}$ & Platinum-cobalt \\
\hline Reuter et al., 1989* & Wire & Baboon & Arterial & Unknown & Unknown & Unknown \\
\hline Zeitlhofer et al., $1990^{*}$ & Unknown & Human (8) & Arterial & Unknown & Unknown & Unknown \\
\hline Nakase et al., 1993* & Wire & Human (2) & Arterial & Unknown & Unknown & Unknown \\
\hline Nakase et al., 1995 & Wire & Human (28) & Arterial & 1 & Diameter: $0.28 \mathrm{~mm}$ & Platinum \\
\hline Stoeter et al., 1995 & Wire & Human (23) & Arterial & 1 & Length: 3-60 mm & Platinum \\
\hline Boniface \& Antoun, 1997 & Wire & Human (2) & Arterial & 1 & Length: 20 mm & Platinum \\
\hline Mikuni et al., 1997 & Wire & Human (7) & Venous & 1 & Length: $30 \mathrm{~mm}$ & Platinum \\
\hline Ishida et al., 1998 & Microwire & Human (2) & Arterial & Unknown & Unknown & Unknown \\
\hline Thömke et al., 1998 & Catheter & Human & Arterial & 16 & Length: $0.5 \mathrm{~mm}$ & Unknown \\
\hline García-Asensio et al., 1999 & Wire & Human (30) & Arterial & 1 & Length: $30 \mathrm{~mm}$ & Platinum \\
\hline Kunieda et al., 2000 & Wire & Human (5) & Venous & 1 & Length: 30 mm & Platinum \\
\hline Llinás et al., 2005 & Nanowire & Unknown & Capillary & $1-4$ & Diameter: 0.0006 mm & Platinum black \\
\hline Watanabe et al., 2009 & Nanowire & Frog & Arterial & Unknown & Diameter: $0.001 \mathrm{~mm}$ & Platinum black \\
\hline Bower et al., 2013† & Depth & Pig & Venous & $4+16$ & $\begin{array}{l}\text { Length (4): } 1 \mathrm{~mm} \text {; diameter } \\
\text { (16): } 0.04 \mathrm{~mm}\end{array}$ & Unknown \\
\hline Oxley et al., 2016 & Stent & Sheep & Venous & 8 & Diameter: $0.75 \mathrm{~mm}$ & Platinum \\
\hline
\end{tabular}

* These articles were not written in English, which limited interpretation.

$\dagger$ Bower et al. used 16 microelectrodes in a 4-contact depth electrode. Only the length of the depth electrodes and the diameter of the microelectrodes were reported.

ritory of the anterior choroidal artery, most likely due to catheter emboli following endovascular Wada testing. ${ }^{2}$

\section{Deep Brain Stimulation}

Although it has not yet been achieved, it is possible that DBS could be performed using an endovascular approach. Teplitzky et al. demonstrated the feasibility of an endovascular approach for DBS via computational modeling. This group identified 5 DBS targets with adjacent vasculatures that were at least $1 \mathrm{~mm}$ in intraluminal diameter (anterior nucleus of the thalamus, fornix, nucleus accumbens, subgenual cingulate white matter, and ventral capsule) by modeling the cerebrovascular system. The subgenual cingulate white matter and fornix were further investigated as potential endovascular DBS targets (which were cited to have roles in depression and memory disorders, respectively), and modeling determined that a ring electrode was preferred over a guidewire electrode for endovascular DBS (due to enhanced vessel wall anchoring capabilities, decreased distance from the electrode to the DBS target, and enhanced neural activation). Teplitzky et al. also demonstrated that with a unilateral electrode implant, endovascular DBS was superior to stereotactic DBS in the production of contralateral activation and comparable to stereotactic DBS in neuronal activation. ${ }^{31}$ Further investigation into the stimulation parameters (such as the current levels) and the safety profile of intravascular stimulation is necessary.

\section{Brain-Machine Interfaces}

The endovascular approach is promising as a chronic and minimally invasive technique to achieving a BMI. Despite the advantages of an invasive approach to BMI (electrocorticography) over scalp EEG (e.g., higher signal bandwidth, closer location to the recording target, higher spatial resolution and signal amplitude, and the lack of interference from both electrooculography and electromyography [with the exception of the reference electrode]), ${ }^{36}$ an invasive approach to BMI requires the implantation of a foreign body into the brain parenchyma, ${ }^{33}$ which may result in inflammation. With an increase in the cross-sectional area of the device, there is increased inflammation in the week following implantation ${ }^{29}$ (most likely due to increased parenchymal damage with insertion). ${ }^{24,26}$ Furthermore, chronic inflammation in the 6 weeks following implantation is independent of device size, and it is believed that increasingly small devices will not circumvent inflammation in its entirety. ${ }^{29}$ An endovascular approach may remedy this problem, as trauma to the brain parenchyma is not associated with electrode implantation.

An endovascular approach to BMI would be advantageous over current invasive approaches. Invasive intracranial subdural electrodes are limited to recording only in the space over which they are implanted (which may lead to limited analyses). ${ }^{12}$ The cerebrovascular system, however, provides a minimally invasive channel to the area superficial to the sensorimotor cortex, allowing for an endovascular, minimally invasive approach to BMI. ${ }^{22}$

\section{Conclusions}

Just as cardiothoracic surgery progressed from openheart surgery with the development of minimally invasive endovascular techniques, neurosurgery may follow the same course. ${ }^{5}$ Cardiac electrophysiology underwent developments in electrode techniques similar to those in neuro- 


$\begin{array}{llc} & \text { CAVERNOUS } & \text { VENOUS } \\ \text { FIRST WIRE } & \text { SINUS } & \text { CATHETER } \\ \text { RECORDING } & \text { RECORDINGS } & \text { RECORDING }\end{array}$
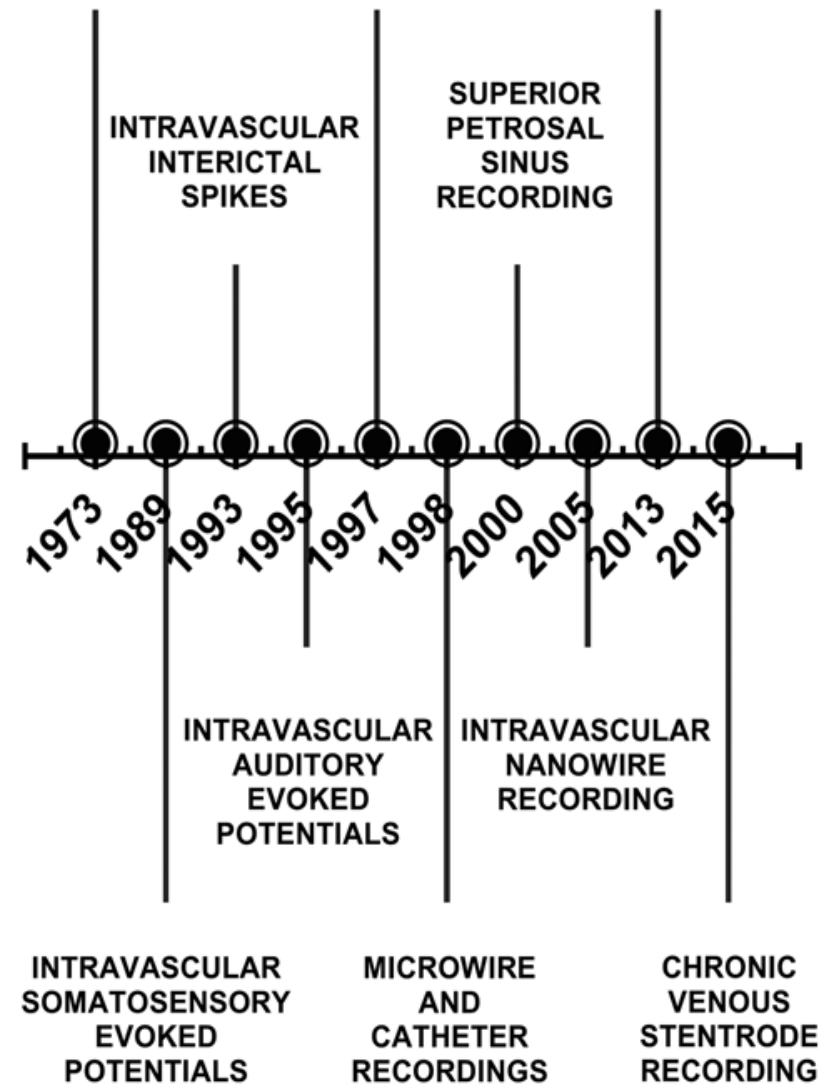

FIG. 3. Historical timeline of the advances in endovascular recording.

surgery, with progression from the external surface leads in the early 1900 s to intracardiac recordings obtained via intravascular catheters in the 1960 s. $^{18}$ In cardiology, the development of endocardial catheter mapping provided localizing information regarding arrhythmogenic foci in the heart for subsequent ablation..$^{15}$ Likewise, in neurosurgery, minimally invasive endovascular techniques may become a tool for the evaluation of epileptogenic foci. . $, 16,19,22$ In cardiology, catheter ablation proved to be efficacious in curing cardiac arrhythmia,${ }^{35}$ and it has been proposed that catheter ablation may be possible in epilepsy surgery. ${ }^{2}$ The development of smaller, chronically implanted cardiac defibrillators that were efficacious for treating ventricular fibrillation and ventricular tachycardia became the standard in cardiac electrophysiology:15 in the same way, chronically implanted electrodes for the prediction and treatment of epilepsy that were deposited endovascularly may become a tool in neurosurgery. An intravenous vagal nerve stimulator placed in the superior vena cava is currently being investigated in cardiology.,13 This device has been shown to decrease left ventricular end-diastolic pressure, decrease the size of an infarction, increase left ventricular ejection fraction at 1 month following induced coronary ischemia, ${ }^{3}$ and prevent ischemia-induced ventricular arrhythmias in dogs.$^{13}$ Furthermore, the efficacy of an intravenous phrenic nerve stimulator for the treatment of patients with central sleep apnea is currently being investigated in a randomized controlled trial, ${ }^{6}$ and a prior nonrandomized study showed a reduction in the apnea-hypopnea index scores by $55 \%$ at 3 months after the initiation of treatment. ${ }^{1}$ Similar advances in neural endovascular stimulation could lead to further advancements in epilepsy management, DBS, and BMI applications.

Endovascular recording technology has advanced from the first wire recording in $1973,{ }^{23}$ to the development of microwire and nanowire recordings in $1998^{14}$ and $2005,{ }^{17}$ respectively, catheter recordings in $1998,{ }^{32}$ and the stentrode in $2015 .{ }^{22}$ With advances in device technology, there was a transition from the use of single unipolar electrodes ${ }^{4,9,16,19,21,28}$ to the use of electrode arrays. . $^{5,17,22,32}$ There has also been an increase in the number of recordings in patients, ${ }^{9}$ but nanowires and stentrodes have yet to be investigated in humans. Furthermore, progress has been made in the ability to chronically record from an intravenous approach. ${ }^{16,19,22}$

The possible future applications of endovascular EEG are numerous. Endovascular EEG could be used in the preoperative evaluation of patients for epilepsy surgery, ${ }^{5,16,19,22}$ or even in the determination of resection margins that could possibly be treated endovascularly. ${ }^{2}$ In addition, computational modeling has demonstrated the feasibility of an endovascular approach to DBS, ${ }^{31}$ and the ability to chronically record in the superior sagittal sinus superficial to the sensorimotor cortex ${ }^{22}$ may lead to the achievement of a minimally invasive BMI.

In order for the use of endovascular EEG to be translated from research to clinical practice, further work is required. Current endovascular EEG recording devices will first need to be translated to humans in the case of nanowires and stentrodes, and then evaluated to determine device superiority. The safety profile will need to be determined for the endovascular ablation of seizure foci, endovascular stimulation in DBS, and the stenting of the superior sagittal sinus for BMI. In addition, further research is necessary to invent retrievable neural endovascular recording devices that would both mitigate the risk of venous infarction and stroke and eliminate the need for chronic anticoagulation. Stimulation parameters will also need to be determined to achieve minimally invasive DBS, and the maximum number of electrodes that can be used to achieve minimally invasive BMI will need to be determined. Further investigation into a wireless recording and stimulation device that is unsusceptible to the wire fatigue previously noted in chronic endovascular recordings ${ }^{22}$ is also needed. With increasingly less invasive approaches to the recording of intracranial EEG, we will see advances in epilepsy surgery, DBS, and BMI.

\section{Acknowledgments}

Ms. Sefcik was supported, in part, by the National Center for Advancing Translational Sciences of the National Institutes of Health under award no. TL1TR001434.

\section{References}

1. Abraham WT, Jagielski D, Oldenburg O, Augostini R, 
Krueger S, Kolodziej A, et al: Phrenic nerve stimulation for the treatment of central sleep apnea. JACC Heart Fail 3:360-369, 2015

2. Ammerman JM, Caputy AJ, Potolicchio SJ: Endovascular ablation of a temporal lobe epileptogenic focus - a complication of Wada testing. Acta Neurol Scand 112:189191,2005

3. Arimura T, Saku K, Kakino T, Nishikawa T, Tohyama T, Akashi T, et al: Intravascular vagal nerve stimulation in acute myocardial infarction markedly reduced infarct size and improved cardiac function in the long term. Circulation 132:A13813, 2015 (Abstract)

4. Boniface SJ, Antoun N: Endovascular electroencephalography: the technique and its application during carotid amytal assessment. J Neurol Neurosurg Psychiatry 62:193-195, 1997

5. Bower MR, Stead M, Van Gompel JJ, Bower RS, Sulc V, Asirvatham SJ, et al: Intravenous recording of intracranial, broadband EEG. J Neurosci Methods 214:21-26, 2013

6. Costanzo MR, Augostini R, Goldberg LR, Ponikowski P, Stellbrink C, Javaheri S: Design of the remede system pivotal trial: a prospective, randomized study in the use of respiratory rhythm management to treat central sleep apnea. J Card Fail 21:892-902, 2015

7. Driller J, Hilal SK, Michelsen WJ, Sollish B, Katz B, Konig W Jr: Development and use of the POD catheter in the cerebral vascular system. Med Res Eng 8:11-16, 1969

8. Duker AP, Espay AJ: Surgical treatment of Parkinson disease: past, present, and future. Neurol Clin 31:799-808, 2013

9. García-Asensio S, Guelbenzu S, Barrena R, Valero P: Technical aspects of intra-arterial electroencephalogram recording. Interv Neuroradiol 5:289-300, 1999

10. Goldring S, Gregorie EM: Surgical management of epilepsy using epidural recordings to localize the seizure focus. Review of 100 cases. J Neurosurg 60:457-466, 1984

11. Hamer HM, Morris HH, Mascha EJ, Karafa MT, Bingaman WE, Bej MD, et al: Complications of invasive video-EEG monitoring with subdural grid electrodes. Neurology 58:97103,2002

12. Hermes D, Vansteensel MJ, Albers AM, Bleichner MG, Benedictus MR, Mendez Orellana C, et al: Functional MRIbased identification of brain areas involved in motor imagery for implantable brain-computer interfaces. J Neural Eng 8:025007, 2011

13. Inagaki M, Kawada T, Lie M, Zheng C, Sunagawa K, Sugimachi M: Intravascular parasympathetic cardiac nerve stimulation prevents ventricular arrhythmias during acute myocardial ischemia. Conf Proc IEEE Eng Med Biol Soc 7:7076-7079, 2005

14. Ishida S, Shimamoto H, Abe T, Motooka H, Sakurai S, Takayama K, et al: Intracranial EEG recording from intravascular electrodes in patients with temporal lobe epilepsy. Epilepsia 39 (Suppl 5):77, 1998 (Abstract)

15. Josephson ME: Electrophysiology of ventricular tachycardia: a historical perspective. Pacing Clin Electrophysiol 26:2052-2067, 2003

16. Kunieda T, Ikeda A, Mikuni N, Ohara S, Sadato A, Taki W, et al: Use of cavernous sinus EEG in the detection of seizure onset and spread in mesial temporal lobe epilepsy. Epilepsia 41:1411-1419, 2000

17. Llinás RR, Walton KD, Nakao M, Hunter I, Anquetil PA: Neuro-vascular central nervous recording/stimulating system: Using nanotechnology probes. J Nanopart Res 7:111-127, 2005

18. Lüderitz B: Clinical and interventional electrophysiology: a personal historical perspective. J Interv Card Electrophysiol 1:243-254, 1997

19. Mikuni N, Ikeda A, Murao K, Terada K, Nakahara I, Taki
W, et al: "Cavernous sinus EEG": a new method for the preoperative evaluation of temporal lobe epilepsy. Epilepsia 38:472-482, 1997

20. Nakase H, Ohnishi H, Touho H, Furuoka N, Yamada K, Takaoka M, et al: [Spike discharge detected by intra-arterial electroencephalography from intra-arterial guide wire in temporal lobe epilepsy.] No To Shinkei 45:973-977, 1993 (Jpn)

21. Nakase H, Ohnishi H, Touho H, Karasawa J, Yamamoto S, Shimizu K: An intra-arterial electrode for intracranial electro-encephalogram recordings. Acta Neurochir (Wien) 136: $103-105,1995$

22. Oxley TJ, Opie NL, John SE, Rind GS, Ronayne SM, Wheeler TL, et al: Minimally invasive endovascular stent-electrode array for high-fidelity, chronic recordings of cortical neural activity. Nat Biotechnol 34:320-327, 2016

23. Penn RD, Hilal SK, Michelsen WJ, Goldensohn ES, Driller J: Intravascular intracranial EEG recording. Technical note. J Neurosurg 38:239-243, 1973

24. Prasad A, Xue QS, Sankar V, Nishida T, Shaw G, Streit WJ, et al: Comprehensive characterization and failure modes of tungsten microwire arrays in chronic neural implants. J Neural Eng 9:056015, 2012

25. Reuter BM, Brassel F, Linke DB, Hartmann A, Kurthen M: [Depth EEG and evoked potentials using an intravascular catheter electrode in the baboon.] EEG EMG Z Elektroenzephalogr Elektromyogr Verwandte Geb 20:8183, 1989 (Ger)

26. Saxena T, Karumbaiah L, Gaupp EA, Patkar R, Patil K, Betancur M, et al: The impact of chronic blood-brain barrier breach on intracortical electrode function. Biomaterials 34:4703-4713, 2013

27. Shimizu H, Suzuki I, Ishizima B: Determination of precise focus distribution and tailored lobectomy in temporal lobe epilepsy. Stereotact Funct Neurosurg 54-55:46-50, 1990

28. Stoeter P, Dieterle L, Meyer A, Prey N: Intracranial electroencephalographic and evoked-potential recording from intravascular guide wires. AJNR Am J Neuroradiol 16:1214-1217, 1995

29. Szarowski DH, Andersen MD, Retterer S, Spence AJ, Isaacson M, Craighead HG, et al: Brain responses to micromachined silicon devices. Brain Res 983:23-35, 2003

30. Taussig D, Montavont A, Isnard J: Invasive EEG explorations. Neurophysiol Clin 45:113-119, 2015

31. Teplitzky BA, Connolly AT, Bajwa JA, Johnson MD: Computational modeling of an endovascular approach to deep brain stimulation. J Neural Eng 11:026011, 2014

32. Thömke F, Stoeter P, Stader D: Endovascular electroencephalography during an intracarotid amobarbital test with simultaneous recordings from 16 electrodes. J Neurol Neurosurg Psychiatry 64:565, 1998

33. Uejima T, Kita K, Fujii T, Kato R, Takita M, Yokoi H: Motion classification using epidural electrodes for lowinvasive brain-machine interface. Conf Proc IEEE Eng Med Biol Soc 2009:6469-6472, 2009

34. Watanabe H, Takahashi H, Nakao M, Walton K, Llinás RR: Intravascular neural interface with nanowire electrode. Electron Commun Jpn 92:29-37, 2009

35. Wellens HJ: Cardiac arrhythmias: the quest for a cure: a historical perspective. J Am Coll Cardiol 44:1155-1163, 2004

36. Wilson JA, Felton EA, Garell PC, Schalk G, Williams JC: ECoG factors underlying multimodal control of a braincomputer interface. IEEE Trans Neural Syst Rehabil Eng 14:246-250, 2006

37. Zeitlhofer J, Feldner G, Heimberger K, Mayr N, Samec P: [Transarterial EEG in superselective cerebral angiography.] EEG EMG Z Elektroenzephalogr Elektromyogr Verwandte Geb 21:70-72, 1990 (Ger) 


\section{Disclosures}

The authors report the following. Drs. Opie and Oxley own shares in SmartStent. Dr. Mocco owns shares in Blockade Medical and Medina and is a consultant for Lazarus Effect, Reverse Medical, Pulsar, Edge Therapeutics, and Medina.

\section{Author Contributions}

Conception and design: Sefcik, Mocco, Oxley. Acquisition of data: Sefcik, Opie, Oxley. Analysis and interpretation of data: Sefcik, Opie, John, Oxley. Drafting the article: Sefcik, Oxley. Critically revising the article: Sefcik, Opie, John, Oxley. Reviewed submitted version of manuscript: all authors. Administrative/technical/material support: Sefcik, John. Study supervision: Oxley.

\section{Correspondence}

Roberta K. Sefcik, Department of Medical Education, Icahn School of Medicine at Mount Sinai, 50 E. 98th St., 7C-1, New York, NY 10029. email: roberta.sefcik@mssm.edu. 